\title{
Upregulated plasmacytoma variant translocation 1 promotes cell proliferation, invasion and metastasis in colorectal cancer
}

\author{
CHAOYU WANG ${ }^{1}$, XIN ZHU $^{1}$, CHIBIN PU $^{2}$ and XUAN SONG ${ }^{1}$ \\ ${ }^{1}$ Department of General Surgery, People's Hospital of Guizhou Province, Guiyang, Guizhou 550002; \\ ${ }^{2}$ Department of Gastroenterology, Zhongda Hospital, Southeast University, Nanjing, Jiangsu 210009, P.R. China
}

Received October 5, 2017; Accepted February 27, 2018

DOI: $10.3892 / \mathrm{mmr} .2018 .8669$

\begin{abstract}
Emerging evidence indicates that the long non-coding RNA (lncRNA) plasmacytoma variant translocation $1(P V T 1)$ is associated with tumourigenesis in various types of cancer. However, its specific effects on the proliferation, invasion and metastasis of colorectal cancer (CRC) are still poorly understood. The present study aimed to investigate PVT1 expression in CRC and explore its role in CRC pathogenesis. The reverse transcriptase-quantitative polymerase chain reaction (RT-qPCR) technique was used to assess PVT1 expression in CRC cell lines. Gene Expression Omnibus (GEO) database analysis and measurement of clinical samples was used to analyse the correlation between PVT1 expression, CRC metastasis and overall survival (OS). In addition, knockdown of $P V T 1$ expression was performed using short interfering RNA (siRNA) and RT-qPCR, western blotting, CCK-8 assays, tumour cell clone-formation and Matrigel invasion assays were used to observe its biological functions in HCT116 cells. The present study demonstrated that the expression of PVT1 in CRC cell lines was higher than that in normal colon mucosal cell lines. Using GEO database analysis and the measurement of clinical samples, it was revealed that CRC patients with high PVTl expression demonstrated poor OS. Multivariate analysis indicated that high $P V T 1$ expression is an independent risk factor for patients with CRC. In addition, PVT1 knockdown suppressed the proliferation, invasion and metastasis of CRC cells in vitro, which were associated with decreasing vimentin, cyclin D1 and cyclin-dependent kinase 4 expression and enhanced E-cadherin expression. The results of the present study suggest that PVT1 may serve a critical role in CRC progression and metastasis and may serve as a potential prognostic biomarker for CRC.
\end{abstract}

Correspondence to: Dr Xuan Song, Department of General Surgery, People's Hospital of Guizhou Province, 83 East Zhongshan Road, Guiyang, Guizhou 550002, P.R. China

E-mail: 1147273943@qq.com

Key words: plasmacytoma variant translocation 1, colorectal cancer, tumourigenesis, invasion, survival

\section{Introduction}

Colorectal cancer (CRC) has a substantial effect on human health; it is the third most prevalent type of cancer worldwide, causing an estimated 693,900 deaths in $2012(1,2)$. Despite the development of new therapeutic approaches and treatments for CRC in recent decades, there has been little change in the overall mortality rate $(3,4)$. CRC development involves a multistep process, which can be due to genetic or environmental factors, leading to mutations in a series of molecules associated with cancer cell proliferation, apoptosis, and differentiation $(5,6)$. Owing to the rising popularity of molecular therapies, various studies have investigated the molecular pathogenesis of CRC by analysing the molecular abnormalities in CRC progression (7-9).

Long non-coding RNAs (lncRNAs) are a subset of RNAs first identified in eukaryotes. They have a transcript length of 200-100,000 nt and lack a complete functional open reading frame (ORF). Rarely, they may encode a short functional peptide and are located in the nucleus or cytoplasm $(10,11)$. Recently, more focus has been placed on IncRNAs for their effect on biological cell behaviour, especially in tumour cells. Increasing number of studies have revealed that lncRNAs are abnormally expressed in many different cancers, such as gastric cancer (12), cervical cancer (13), non-small cell lung cancer (NSCLC) (14), and CRC (15). These abnormally expressed IncRNAs have been used as biomarkers for cancer therapies and diagnoses.

The IncRNA plasmacytoma variant translocation 1 (PVTI) is located on chr8q24.21 and is 1,716 nt in length. The gene region of $P V T 1$ contains the myelocytomatosis (myc) oncogene; the MYC protein can result in the accumulation of $P V T I$ in primary human cancers (16). Emerging evidence indicates that $P V T 1$ is associated with tumourigenesis in various cancers, including gastric cancer (17), NSCLC (18), and hepatocellular cancer (19); however, the specific effects of PVTI on the proliferation, invasion, and metastasis of CRC are still poorly understood. In the present study, we first demonstrated that $P V T 1$ is overexpressed in CRC tissues and cell lines. We then determined that CRC patients with high PVTI expression showed poor overall survival (OS), by analysing Gene Expression Omnibus (GEO) datasets. PVTI knockdown was also shown to suppress the proliferation, invasion, and metastasis of CRC cells in vitro. These results suggest that PVTI 
plays a significant role in CRC tumourigenesis and tumour progression.

\section{Materials and methods}

Bioinformatics analysis. All microarray expression dates, containing primary CRC data and their correlated clinic data, were deposited in the GEO database: GSE9348 (20), GSE23878 (21), GSE22598 (22), and GSE17536 (23) (Affymetrix Human Genome U133 Plus 2.0 platform) and GSE50760 (24) (Illumina HiSeq 2000 platform). GSE9348 has 70 primary CRC samples and 12 normal colon samples; GSE23878 has 35 primary CRC samples and 24 normal colon samples; GSE22598 contains 17 pairs of CRC and adjacent non-tumour tissues; GSE17536 is divided into the low PVTI expression group $(\mathrm{n}=83)$ and high $P V T 1$ expression group $(n=60)$; GSE50760 has 17 metastasis CRC samples and 37 non-metastasis CRC samples.

Cell culture and transfection. The human colorectal cancer cell lines used in this study were obtained from the American Type Culture Collection (ATCC; Manassas, VA, USA). HCT116 cells were maintained in DMEM (Dulbecco's modified Eagle's medium) with $10 \%$ fetal bovine serum (FBS; Invitrogen; Thermo Fisher Scientific, Inc., Waltham, MA, USA), and other cell lines (SW480, HT29, NCM460, SW620, CaCO2) were cultured in RPMI-1640 media (Invitrogen; Thermo Fisher Scientific, Inc.) supplemented with 10\% FBS. HCT116, SW480, HT29, NCM460, SW620 and CaCO2 are all human colorectal cancer cell lines, while NCM460 is a normal colonic epithelial cell line from the tissue of a patient with gastric cancer. Transfection was conducted with. When cell densities were approximately 60\%, $50 \mathrm{nM}$ short interfering RNA (siRNA) oligos were transfected by Lipofectamine 3000 (Invitrogen, USA). The sequences of the PVT1 targeting siRNAs were as follows: PVT1-si-1: 5'-CUGGACCUUAUGGCUCCA-3'; PVT1-si-2: 5'-CACUGAGGCUACUGCAUCU-3'; sequences of non-target scramble controls were provided by RiboBio (Guangzhou, China).

Reverse transcriptase-quantitative polymerase chain reaction $(R T-q P C R)$. Tissue RNA isolation and amplification were performed as described previously (25). RNA was isolated from the cells, using Trizol reagent (Invitrogen, The Netherlands). For the RT-qPCR, RNA was reverse transcribed to cDNA, using a Revert Aid First Strand cDNA Synthesis kit (Fermentas; Thermo Fisher Scientific, Inc.,). RT-qPCR was performed using a SYBR_Premix ExTaq II kit (Takara Biotechnology Co., Ltd., Dalian, China) in the CFX96 Real-Time PCR Detection System (Bio-Rad Laboratories, Inc., Hercules, CA, USA) to determine the relative expression of target genes. The following program was used for qPCR: $95^{\circ} \mathrm{C}$ for $30 \mathrm{sec}$ followed by 40 cycles of $95^{\circ} \mathrm{C}$ for $5 \mathrm{sec}$ and then $60^{\circ} \mathrm{C}$ for $30 \mathrm{sec}$. The sequences of RT-qPCR primers were as follows: PVT1 forward 5'-TTCAGCACTCTG GACGGACTT-3', reverse 5'-TATGGCATGGGCAGGGTA G-3'; human cyclin D1 forward 5'-TCGTTGCCCTCTGTG CCACA-3', reverse 5'-GCAGTCCGGGTCACACTTGA-3'; human E-cadherin forward 5'-TGAAGCCCCCATCTTTGT GC-3', reverse 5'-GGCTGTGTACGTGCTGTTCT-3; human vimentin forward 5'-TGA AGCCAATTGCAGGAGGAG A-3', reverse 5'-TCTTGGCAGCCACACTTTCAT-3'; human cyclin-dependent kinase 4 (CDK4) forward 5'-TTGGTGTCG GTGCCTATGGG-3', reverse 5'-CCATCAGCCGGACAA CATTGGG-3'; human GAPDH forward 5'-AACGGATTT GGTCGTATTGG-3', reverse 5'-TTGATTTTGGAGGGATCT CG-3'.

Western blotting. Cell lysis, cell lysate electrophoresis, and target protein visualisation were performed as described previously (25). Firstly, the cells were resuspended in lysis buffer [1\% Nonidet P-40, $50 \mathrm{mM}$ Tris- $\mathrm{HCl}, \mathrm{pH} 7.5,50 \mathrm{mM} \mathrm{NaF}$, $2 \mathrm{mM}$ EDTA, $400 \mathrm{mM} \mathrm{NaCl}, 10 \%$ glycerol plus Complete protease inhibitor mixture (Merck KGaA, Darmstadt, Germany)]. Then, $50 \mu \mathrm{g}$ of cell lysates were separated by $10 \%$ sodium dodecyl sulphate-polyacrylamide gel electrophoresis (SDS-PAGE) and transferred onto Nitrocellulose membrane (Bio-Rad Laboratories, Inc.). After the membranes were blocked in Tris-buffered saline/Tween-20 (25 mM Tris- $\mathrm{HCl}, 150 \mathrm{mM} \mathrm{NaCl}, \mathrm{pH} 7.5$ and $0.05 \%$ Tween-20) with $5 \%$ defatted milk for $1 \mathrm{~h}$ at $37^{\circ} \mathrm{C}$, the membranes were incubated overnight at $4^{\circ} \mathrm{C}$ with the primary antibodies, including E-cadherin (cat. no. 3195, 1:1,000; Cell Signaling Technology, Inc., Danvers, MA, USA), vimentin (cat. no. 5741, 1:500; Cell Signaling Technology, Inc.), cyclin D1 (cat. no. 2978, 1:1,000; Cell Signaling Technology, Inc.), CDK4 (cat. no. 12790, 1:500; Cell Signaling Technology, Inc.), and GAPDH (cat. no. 5174, 1:1,000; Cell Signaling Technology, Inc.). After washing with TBST, the membranes were incubated with horseradish peroxidase-conjugated secondary antibodies (Santa Cruz Biotechnology, Inc., Dallas, TX, USA) and visualized using the ECL detection system. Densitometric analysis of immunodetected bands was performed using Image Analysis software (Bio-Rad Laboratories, Inc.).

Cell proliferation assay. The transfected cells were seeded in 96-well flat-bottom plates at a density of $2 \times 10^{3}$ cells/well in $200 \mathrm{ul}$ of medium, and cultured for the CCK-8 (Dojin Laboratories, Kumamoto, Japan), with the operating steps carried out as described previously (26).

Tumour cell clone-formation assay. The tumour cell clone-formation assay was carried out as described previously (26). Briefly, $1 \times 10^{3}$ cells were seeded into each well of a 6-well culture plate and incubated for 14 days, after which the cell colonies were stained with haematoxylin. Then, the clone formation efficiency was calculated. Each experiment was repeated three times independently.

Matrigel invasion assays. Colorectal cell invasiveness was determined in a 24 -well transwell plate ( $8 \mu$ p pore size; Costar), as described previously (26). Briefly, $5 \times 10^{4}$ cells were placed in the upper chamber of each insert coated with $200 \mathrm{mg} / \mathrm{ml}$ of Matrigel (BD Biosciences, Franklin Lakes, NJ, USA). After 48 h, the invaded cells were stained with haematoxylin and counted. Each experiment was repeated three times independently.

Statistical analysis. All statistical analyses were carried out using SPSS version 18.0 (SPSS, Inc., Chicago, IL, USA) and presented with Graphpad prism software (GraphPad 
A

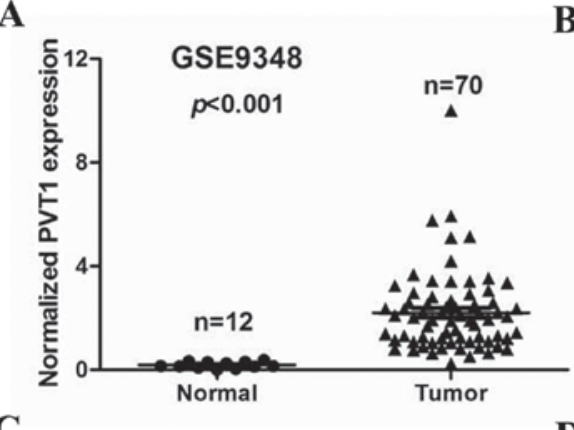

C

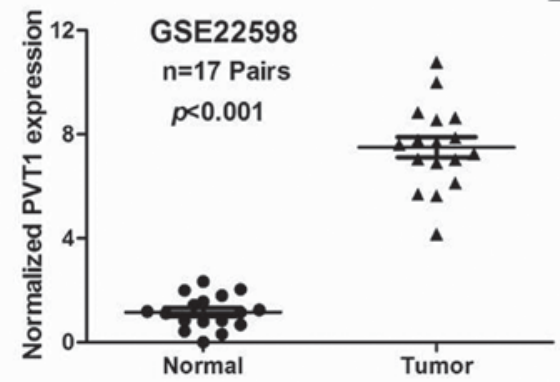

B

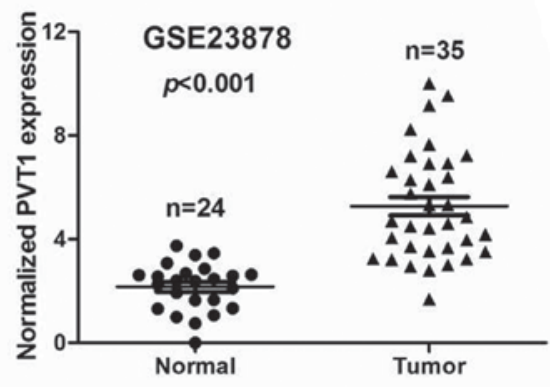

D

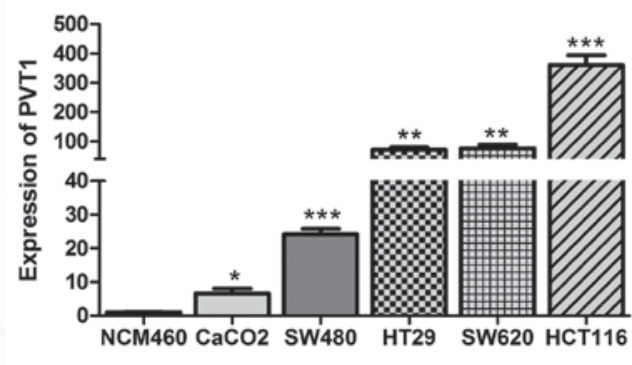

Figure 1. PVT1 is highly expressed in CRC tissues and cell lines. PVT1 expression, as measured by Affymetrix microarray, was upregulated in CRC tissues compared with that in normal colon mucosal tissues in (A) \#GSE9348 (containing 12 normal colorectal tissues and 70 CRC tissue biopsies), (B) \#GSE23878 (containing 24 normal colorectal tissues and 35 CRC tissue biopsies) and (C) \#GSE22598 (containing 17 pairs of CRC tissues and corresponding normal colorectal tissues) from the GEO database. (D) PVT1 expression significantly increased in CRC cell lines (SW480, HT29, Caco-2, HCT116, and SW620) compared with that in NCM460, a normal colon mucosal cell line. Data are shown as mean \pm standard error of the mean. ${ }^{*} \mathrm{P}<0.05,{ }^{* *} \mathrm{P}<0.01,{ }^{* * *} \mathrm{P}<0.001$ vs. NCM460 cells. PVT1, plasmacytoma variant translocation 1; GEO, Gene Expression Omnibus; CRC, colorectal cancer.

Software, Inc., La Jolla, CA, USA). Data are expressed as the mean \pm standard error of the mean. Differences between two independent groups were tested using Student's t-test and analysis of variance was performed for comparisons among multiple groups. OS was calculated using the Kaplan-Meier method, and the results of the analysis were considered significant in a log-rank test if $\mathrm{P}<0.05$.

\section{Results}

PVT1 is upregulated in CRC tissues and cell lines. To determine the expression of PVT1 in CRC tissues, we first analysed three previously published datasets (nos. GSE9348, GSE23878, and GSE22598), using Affymetrix HG_U133 Plus 2 arrays to identify dysregulated lncRNAs in CRC tissues. We found that the IncRNA $P V T 1$ was significantly up regulated in GSE9348, GSE23878, and GSE22598 (P<0.001, Fig. 1A-C). Next, PVT1 expression was also determined by RT-qPCR in five CRC cell lines (SW480, HT29, Caco-2, HCT116, and SW620), and the normal colon mucosal cell line NCM460. The results showed that PVT1 expression was higher in the CRC cell lines than in NCM460 $(\mathrm{P}<0.05$, Fig. 1D), and that PVT1 expression was the highest in HCT116 cells. Therefore, we selected HCT116 cells for further studies.

Upregulation of PVT1 predicts a poor prognosis and could be regarded as an independent predictor for $O S$ in $C R C$. We next assessed the correlation between $P V T 1$ expression and distant metastasis in CRC tissues. We analysed a previously published dataset (no. GSE50760), using Illumina HiSeq 2000 arrays, to identify dysregulated lncRNAs in CRC. We found that the higher expression of PVT1 was significantly correlated with CRC distant metastasis $(\mathrm{P}<0.001$, Fig. 2A).

To assess the prognostic value of $P V T 1$ expression in CRC patients, the SynTarget database (27,28)-a database for survival analyses in cancer-was used to analyse the clinical impact of PVT1 expression patterns on the survival of CRC patients in a CRC specimen expression profile dataset (no. GSE17536). The results revealed that $P V T 1$ expression showed a negative correlation with the OS of CRC patients ( $\mathrm{P}=0.0296$, Fig. 2B). Collectively, these data indicate that high PVT1 expression is an independent risk factor for CRC patients.

Knockdown of PVT1 inhibits cell proliferation and invasion in $C R C$. To verify the function of $P V T 1$ in colon cancer cells, we first measured the efficiency of the siRNA si-PVT1. Compared with the siPVT1-1 and siPVT1-2 groups, the siPVT1-1+2 group showed the highest efficiency in HCT116 cells (Fig. 3A). Therefore, we chose siPVT1-1+2 for in vivo knockdown of $P V T 1$ expression to assess the biological function of $P V T 1$ in CRC tissues. We investigated the effect of PVT1 knockdown on $\mathrm{CRC}$ cell proliferation by performing $\mathrm{CCK}-8$ proliferation assays. PVT1 knockdown expression significantly inhibited HCT116 cell proliferation compared to that of the control group in the $96 \mathrm{~h}(\mathrm{P}<0.001$, Fig. 3B). PVT1 knockdown also inhibited HCT116 cell clone formation compared to that of the control group $(\mathrm{P}<0.05$, Fig. $3 \mathrm{C})$.

We also explored the effect of PVT1 knockdown on CRC cell invasion. A transwell invasion assay was performed to assess the effect of PVT1 on the invasiveness of CRC cells. PVT1 knockdown significantly inhibited HCT116 cell invasion compared to that of the control group $(\mathrm{P}<0.01$, Fig. 4$)$. These results demonstrated that $P V T 1$ knockdown suppressed 
A

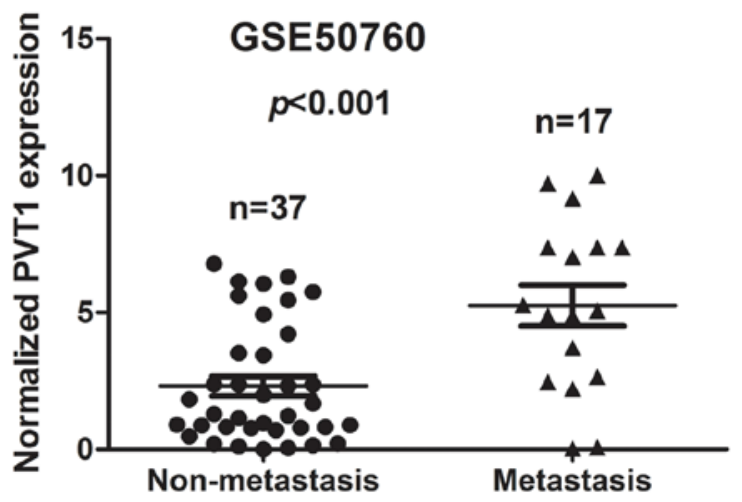

B

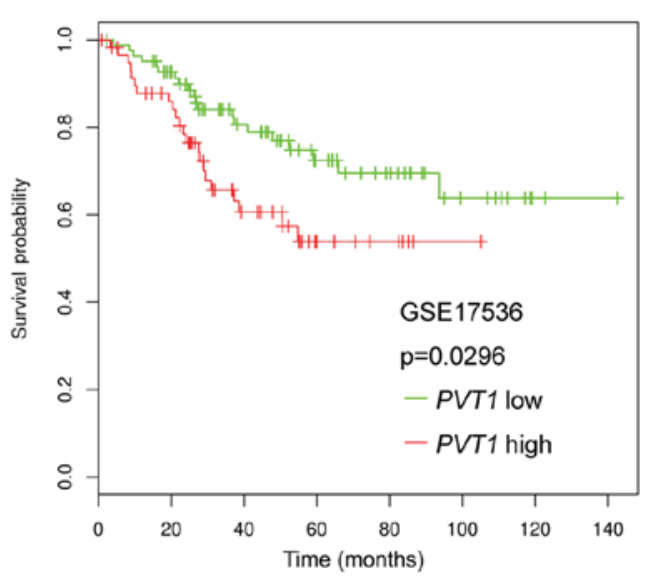

Figure 2. Association between $P V T 1$ expression and clinicopathological features. (A) Relative expression of $P V T 1$ in non-metastasis and metastasis human CRC tissues were obtained from the GEO database (no. GSE50760, non-metastasis, n=37; metastasis, n=17). (B) SynTarget database was used to analyse the clinic impact of $P V T 1$ expression patterns on CRC patient's survival in a CRC specimen expression profile dataset (no. GSE17536, the specimen of GSE17536 was divided into two groups, using the SynTarget database: Group 1, low expression of $P V T 1, \mathrm{n}=83$; group 2, high expression of $P V T 1, \mathrm{n}=60$ ). $P V T 1$, plasmacytoma variant translocation 1; GEO, Gene Expression Omnibus; CRC, colorectal cancer.

$\mathbf{A}$

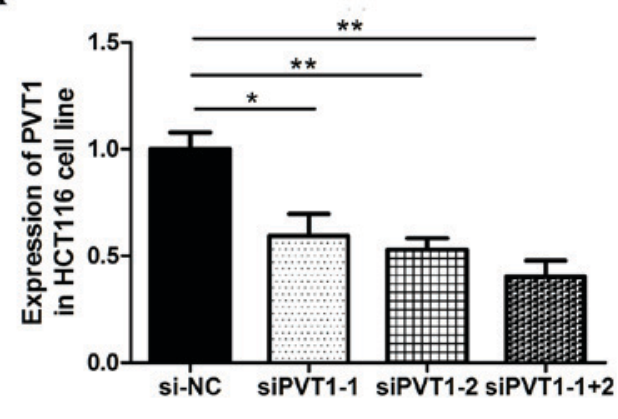

B

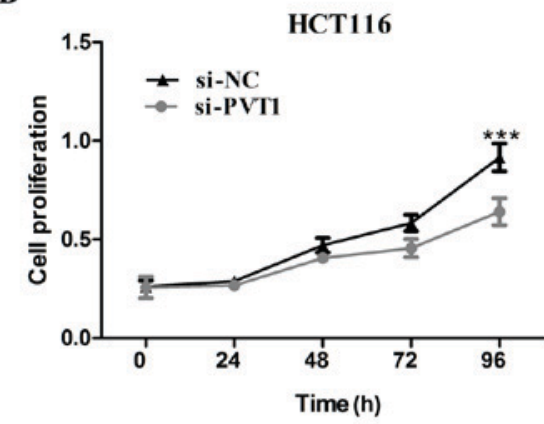

C
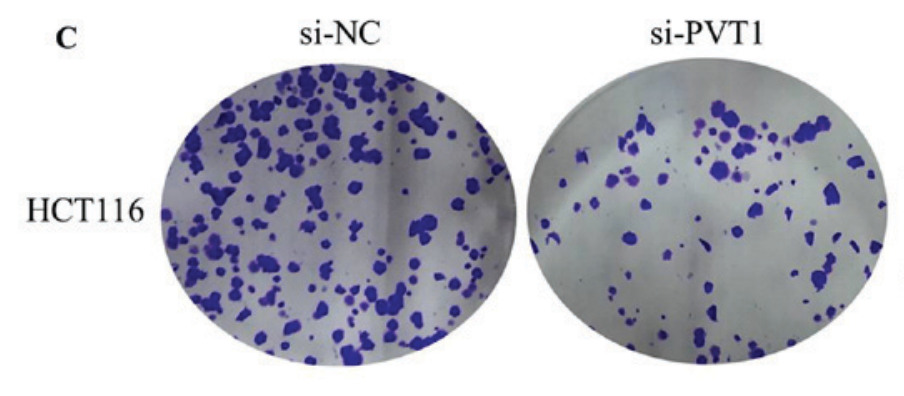

si-PVT1

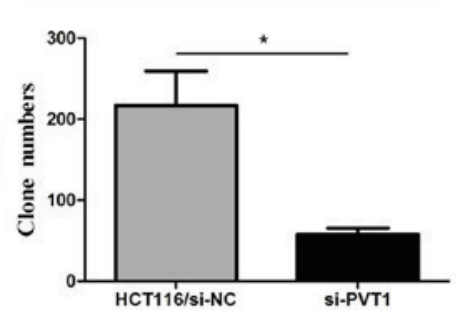

Figure 3. Knocking down $P V T 1$ expression inhibits proliferation of colorectal cancer cells. (A) Interference efficiency of si- $P V T 1$ was verified in HCT116 cells. HCT116 cells were transfected with either si-NC or si-PVT1 $(1 \#, 2 \#, 1+2 \#)$ for $48 \mathrm{~h}$, and then $P V T 1$ expression was analysed by reverse transcription-quantitative polymerase chain reaction. (B and C) CCK-8 assay and cell clone-formation assay was used to detect the cell proliferative ability after transfecting with si-NC or si-PVT1 for $48 \mathrm{~h}$ in HCT116 cells. Data are shown as the mean \pm standard error of the mean. ${ }^{*} \mathrm{P}<0.05,{ }^{* * *} \mathrm{P}<0.01$ and ${ }^{* * * *} \mathrm{P}<0.001 \mathrm{vs}$. si-PVT1 group at $96 \mathrm{~h}$. PVT1, plasmacytoma variant translocation 1; NC, negative control; si, short interfering.

the proliferation, invasion, and metastasis of CRC cells in vitro.

Knockdown of PVT1 suppresses proliferation and EMT markers in CRC. To confirm that PVTl knockdown suppresses the proliferation, invasion, and metastasis of CRC cells in vitro, RT-qPCR and western blotting were used to assess the mRNA and protein level of the epithelial marker E-cadherin, mesenchymal markers vimentin, and proliferation markers cyclin D1 and CDK4 in HCT116 cell lines. PVT1 knockdown significantly decreased vimentin and enhanced E-cadherin expression ( $\mathrm{P}<0.05$, Fig. 5A-B), thereby inhibiting the progression of EMT. Meanwhile, PVT1 knockdown significantly inhibited cyclin D1 and CDK4 ( $\mathrm{P}<0.01$, Fig. 5C and D). This indicates that $P V T 1$ regulates proliferation and EMT markers expression in CRC cell lines.

\section{Discussion}

$\mathrm{CRC}$ is one of the most common causes of cancer-associated mortality worldwide (29), especially in developed countries. It wasestimated in 2015 that there were 777,987 new cases 

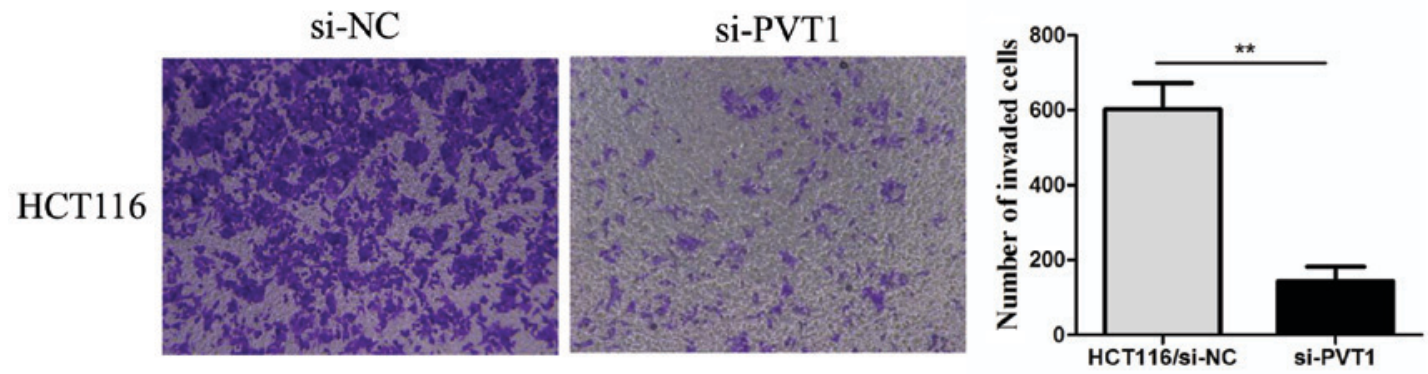

Figure 4. Knocking down $P V T 1$ expression inhibits invasion of colorectal cancer cells. Cell invasive ability was determined by transwell Matrigel assay after transfecting with si-NC or si-PVT1 for $48 \mathrm{~h}$ in HCT116 cells. Data are shown as the mean \pm standard error of the mean. ${ }^{* * *} \mathrm{P}<0.01$. $P V T 1$, plasmacytoma variant translocation 1; NC, negative control; si, short interfering.

A

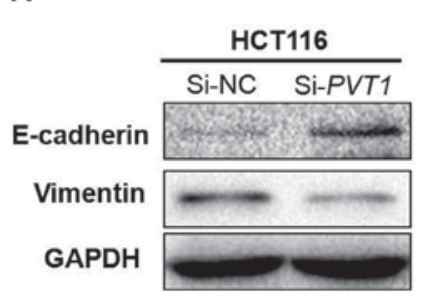

C

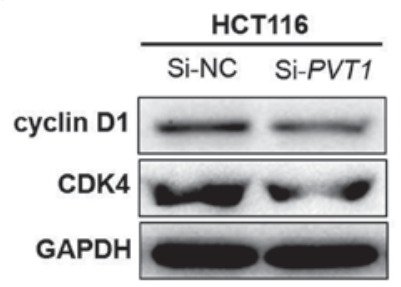

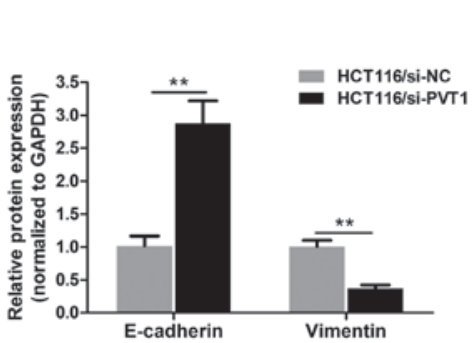

B
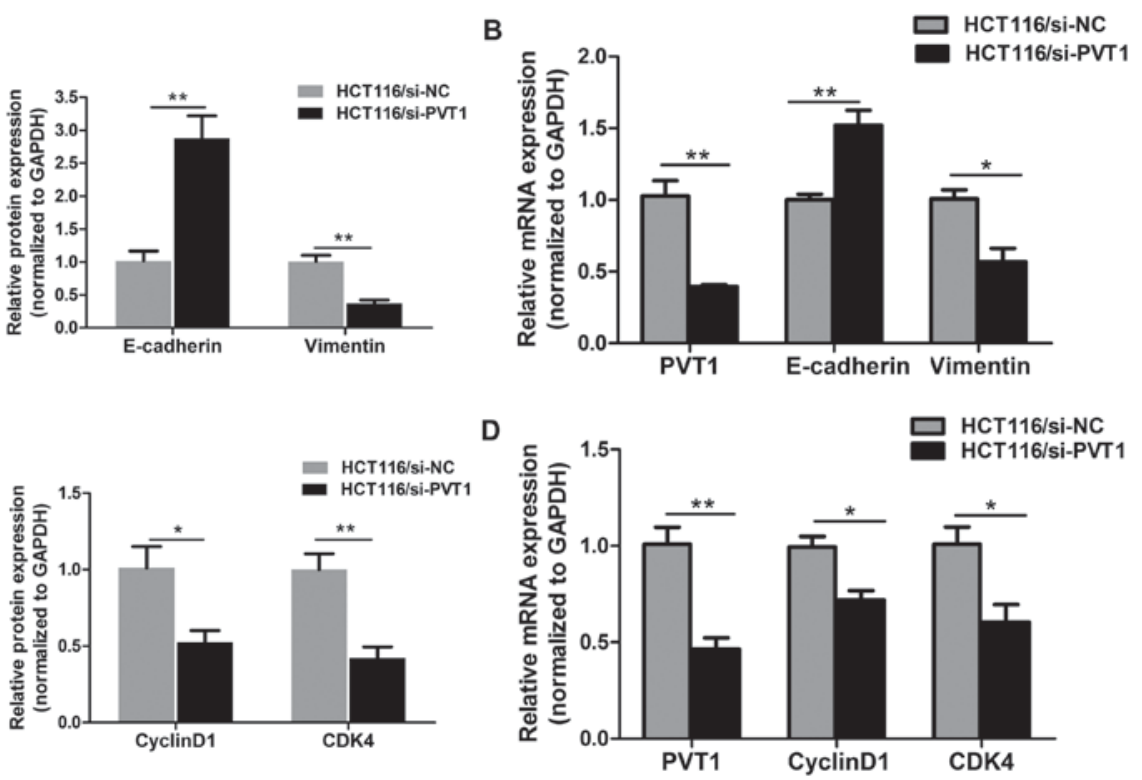

Figure 5. Knockdown of $P V T 1$ suppresses proliferation and EMT markers in CRC. (A) Protein and (B) mRNA expressions of E-cadherin and vimentin in CRC cells were analysed by western blot analysis and RT-qPCR, respectively, following transfection with si-NC or si-PVT1 for $48 \mathrm{~h}$ in HCT116 cells. (C) Protein and (D) mRNA expression of cyclin D1 and CDK4 in CRC cells were analysed by western blot analysis and RT-qPCR following transfection with si-NC or si-PVT1 for $48 \mathrm{~h}$ in HCT116 cells. Data are shown as the mean \pm standard error of the mean. ${ }^{*} \mathrm{P}<0.05,{ }^{* *} \mathrm{P}<0.01$. RT-qPCR, reverse transcription-quantitative polymerase chain reaction; PVT1, plasmacytoma variant translocation 1; CRC, colorectal cancer; NC, negative control; si, short interfering; EMT, epithelial-mesenchymal transition; CDK4, cyclin dependent kinase 4; si, small interfering.

and 352,589 deaths from CRC in developed countries (30). Surgery is currently the primary method of treatment for CRC, along with adjuvant radio-chemotherapy treatments. Although substantial progress has been made in the diagnosis and treatment of CRC, it retains a high morbidity and mortality rate owing to frequent recurrence and metastasis after treatment. Therefore, the treatment of $\mathrm{CRC}$ requires a novel therapeutic target to better control recurrence and metastasis.

LncRNAs are emerging as pivotal regulators in various biological processes. They are modulators of gene expression at the epigenetic, transcriptional, and post-transcriptional levels $(31,32)$, controlling the fate of cellular processes including cell proliferation, apoptosis, and differentiation (33). Recent studies have revealed that disrupting or disabling lncRNA expression strongly correlates with a decrease in the incidence and development of malignant tumours, because lncRNAs have roles in cancer cell proliferation, the epithelial-mesenchymal transition (EMT), and drug resistance (34,35). Because lncRNAs are easier to extract, can be detected with higher specificity and sensitivity, and exist steadily in the blood and tissue (36), they have great potential to be a novel biomarker for cancer diagnosis, predicting recurrence, and chemosensitivity. Several lncRNAs have been shown to be differentially expressed in CRC and indicators of a poor prognosis, including MEG3 (37), GAS5 (38), MALATl (39), TUG1 (40), HOTAIR (41), and PVT1 (42).

The lncRNA $P V T 1$ is reported to be overexpressed in many diseases, including several cancers. PVT1 overexpression has recently been identified as an independent predictor for OS in various human cancers, such as gastric cancer (17), NSCLC (18), and hepatocellular cancer (19). However, there has been insufficient research on the association of PVT1 expression with the OS of CRC patients. Takahashi et al (42) demonstrated that the location of PVT1 was similar to that of $M Y C$, which were mapped to chromosome 8q24. They also showed that $8 \mathrm{q} 24$ copy-number amplification promoted 
MYC and PVTI expression-prognostic indicators for CRC in patients. Similarly, Li et al (43) reported that the higher levels of AFAP1-AS1, MALAT1, H19, HOXA-AS2, BCAR4 or PVT1 in CRC tissues might predict the poor prognosis of CRC patients. In our study, we aimed to explore this IncRNA, whichhas the potential to be developed into a novel biomarker for CRC diagnosis and prognosis. We reported that $P V T 1$ expression was significantly higher in CRC tissues than in normal colon mucosal tissues by GEO database analysis. Furthermore, multivariate analysis showed that CRC patients with PVT1 overexpression had a poorer OS time, which indicates that overexpression of PVT1 may be an independent indicator of poor prognosis in CRC patients.

Evidence strongly suggests that PVT1 plays a critical role in the development and progression of cancer by regulating cancer cell proliferation, metastasis, cell cycle, apoptosis, stemness, and drug resistance (44). Huang et al (45) demonstrated that PVT1 was overexpressed in small cell lung cancer (SCLC) tissues, and that knocking down PVT1 expression with siRNA significantly suppressed SCLC cell migration and invasion in vitro. Additionally, Kong et al (17) revealed that upregulation of PVT1 promotes cell proliferation in gastric cancer by epigenetically regulating p15 and p16. Shen et al (46) also showed that PVT1 could decrease miR-195 expression by enhancing histone H3K27me3 in the $m i R-195$ promoter region and by direct sponging of $m i R-195$ to modulate EMT and chemo-resistance in cervical cancer cells. However, the effects of PVT1 on CRC proliferation, invasion, and metastasis are poorly understood. Guo et al (47) reported that PVT1 may be a new oncogene co-amplified with $c-M y c$ in CRC tissues and functionally correlated with the proliferation and apoptosis of CRC cells. Our results demonstrated that inhibition of PVT1 suppressed CRC cell proliferation, invasion, and metastasis in HCT116 cell lines, which was associated with decreased vimentin, cyclin D1, and CDK4 expression, but enhanced E-cadherin expression. This indicates that PVT1 contributes to the regulation of proliferation and EMT marker expression in CRC cell lines.

In summary, the results presented in this study indicate that PVT1 expression is upregulated in CRC patients, and that patients with high $P V T 1$ expression show poor OS. Multivariate analysis indicated that high $P V T 1$ expression is an independent risk factor for CRC patients. We also demonstrated that PVTI expression mediates the proliferation, invasion, and metastasis of CRC cells. PVT1 knockdown significantly suppressed the proliferative and invasive capabilities of CRC cells. However, our study exists two limitations: 1) our study was limited by the use of only one CRC cell line; 2) our study was not further investigate the regulatory mechanism underlying PVTl's promotion of the proliferation, invasion, and metastasis of CRC cells. Taken together, our study demonstrated the oncogene role of $P V T 1$ in tumour progression of $\mathrm{CRC}$, and shows potential as a target for development of novel CRC therapies after further investigation.

\section{Acknowledgements}

Not applicable.

\section{Funding}

No funding was received.

\section{Availability of data and materials}

The datasets generated and/or analyzed during the current study are available in the Gene Expression Omnibus datasets (ncbi.nlm.nih.gov/gds/).

\section{Authors' contributions}

CW and XS developed the concept and designed the study. $\mathrm{CW}, \mathrm{XZ}$ and $\mathrm{CP}$ collected the data. CW, XZ and CP analysed and interpreted the data. All authors contributed to the writing of the manuscript.

\section{Ethics approval and consent to participate}

Not applicable.

\section{Consent for publication}

Not applicable.

\section{Competing interests}

The authors confirm that they have no competing interests.

\section{References}

1. Chen W, Zheng R, Baade PD, Zhang S, Zeng H, Bray F, Jemal A, Yu XQ and He J: Cancer statistics in China, 2015. CA Cancer J Clin 66: 115-132, 2016.

2. Chen C, Wang L, Liao Q, Huang Y, Ye H, Chen F, Xu L, Ye M and Duan S: Hypermethylation of EDNRB promoter contributes to the risk of colorectal cancer. Diagn Pathol. 8: 199, 2013.

3. Sunkara V and Hebert JR: The colorectal cancer mortality-to-incidence ratio as an indicator of global cancer screening and care. Cancer 121: 1563-1569, 2015.

4. Brenner H, Kloor M and Pox CP: Colorectal cancer. Lancet 383: 1490-1502, 2014

5. Rupnarain C, Dlamini Z, Naicker S and Bhoola K: Colon cancer: Genomics and apoptotic events. Biol Chem 385: 449-464, 2004.

6. Ou C, Sun Z, Li S, Li G, Li X and Ma J: Dual roles of yes-associated protein (YAP) in colorectal cancer. Oncotarget 8: 75727-75741, 2017.

7. Edwards BK, Ward E, Kohler BA, Eheman C, Zauber AG, Anderson RN, Jemal A, Schymura MJ, Lansdorp-Vogelaar I, Seeff LC, et al: Annual report to the nation on the status of cancer, 1975-2006, featuring colorectal cancer trends and impact of interventions (risk factors, screening and treatment) to reduce future rates. Cancer 116: 544-573, 2010.

8. Colussi D, Brandi G, Bazzoli F and Ricciardiello L: Molecular pathways involved in colorectal cancer: Implications for disease behavior and prevention. Int J Mol Sci 14: 16365-16385, 2013

9. Vaiopoulos AG, Athanasoula K and Papavassiliou AG: Epigenetic modifications in colorectal cancer: Molecular insights and therapeutic challenges. Biochim Biophys Acta 1842: 971-980, 2014.

10. Guttman M and Rinn JL: Modular regulatory principles of large non-coding RNAs. Nature 482: 339-346, 2012.

11. Bazzini AA, Johnstone TG, Christiano R, Mackowiak SD, Obermayer B, Fleming ES, Vejnar CE, Lee MT, Rajewsky N, Walther TC and Giraldez AJ: Identification of small ORFs in vertebrates using ribosome footprinting and evolutionary conservation. EMBO J 33: 981-993, 2014.

12. Song H, Sun W, Ye G, Ding X, Liu Z, Zhang S, Xia T, Xiao B, $\mathrm{Xi} Y$ and Guo J: Long non-coding RNA expression profile in human gastric cancer and its clinical significances. J Transl Med 11: 225, 2013. 
13. Shang C, Zhu W, Liu T, Wang W, Huang G, Huang J, Zhao $\mathrm{P}$, Zhao $\mathrm{Y}$ and Yao S: Characterization of long non-coding RNA expression profiles in lymph node metastasis of early-stage cervical cancer. Oncol Rep 35: 3185-3197, 2016

14. Yang Y, Li H, Hou S, Hu B, Liu J and Wang J: The noncoding RNA expression profile and the effect of lncRNA AK126698 on cisplatin resistance in non-small-cell lung cancer cell. PLoS One 8: e65309, 2013.

15. Wu H, Wu R, Chen M, Li D, Dai J, Zhang Y, Gao K, Yu J, Hu G, Guo Y, et al: Comprehensive analysis of differentially expressed profiles of lncRNAs and construction of miR-133b mediated ceRNA network in colorectal cancer. Oncotarget 8: 21095-21105, 2017.

16. Tseng YY, Moriarity BS, Gong W, Akiyama R, Tiwari A, Kawakami H, Ronning $\mathrm{P}$, Reuland B, Guenther K, Beadnell TC, et al: PVT1 dependence in cancer with MYC copy-number increase. Nature 512: 82-86, 2014.

17. Kong R, Zhang EB, Yin DD, You LH, Xu TP, Chen WM, Xia R, Wan L, Sun M, Wang ZX, et al: Long noncoding RNA PVT1 indicates a poor prognosis of gastric cancer and promotes cell proliferation through epigenetically regulating $\mathrm{p} 15$ and $\mathrm{p} 16 . \mathrm{Mo}$ Cancer 14: 82, 2015

18. Cui D, Yu CH, Liu M, Xia QQ, Zhang YF and Jiang WL: Long non-coding RNA PVT1 as a novel biomarker for diagnosis and prognosis of non-small cell lung cancer. Tumour Biol 37: 4127-4134, 2016

19. Wang F, Yuan JH, Wang SB, Yang F, Yuan SX, Ye C, Yang N, Zhou WP, Li WL, Li W and Sun SH: Oncofetal long noncoding RNA PVT1 promotes proliferation and stem cell-like property of hepatocellular carcinoma cells by stabilizing NOP2 Hepatology 60: 1278-1290, 2014.

20. Hong Y, Downey T, Eu KW, Koh PK and Cheah PY: A 'metastasis-prone' signature for early-stage mismatch-repair proficient sporadic colorectal cancer patients and its implications for possible therapeutics. Clin Exp Metastasis 27: 83-90, 2010.

21. Uddin S, Ahmed M, Hussain A, Abubaker J, Al-Sanea N, AbdulJabbar A, Ashari LH, Alhomoud S, Al-Dayel F, Jehan Z, et al: Genome-wide expression analysis of Middle Eastern colorectal cancer reveals FOXM1 as a novel target for cancer therapy. Am J Pathol 178: 537-547, 2011.

22. Okazaki S, Ishikawa $T$, Iida $S$, Ishiguro $M$, Kobayashi $H$, Higuchi T, Enomoto M, Mogushi K, Mizushima $\mathrm{H}$, Tanaka $\mathrm{H}$, et al: Clinical significance of UNC5B expression in colorectal cancer. Int J Oncol 40: 209-216, 2012.

23. Smith JJ, Deane NG, Wu F, Merchant NB, Zhang B, Jiang A Lu P, Johnson JC, Schmidt C, Bailey CE, et al: Experimentally derived metastasis gene expression profile predicts recurrence and death in patients with colon cancer. Gastroenterology 138 958-968, 2010

24. Kim SK, Kim SY, Kim JH, Roh SA, Cho DH, Kim YS and Kim JC: A nineteen gene-based risk score classifier predicts prognosis of colorectal cancer patients. Mol Oncol 8: 1653-1666, 2014.

25. Ou C, Sun Z, Zhang H, Xiong W, Ma J, Zhou M, Lu J, Zeng Z, Bo $\mathrm{X}$, Chen $\mathrm{P}$, et al: SPLUNC1 reduces the inflammatory response of nasopharyngeal carcinoma cells infected with the EB virus by inhibiting the TLR9/NF- $\kappa$ B pathway. Oncol Rep 33 : 2779-2788, 2015

26. Ou C, Sun Z, Li X, Li X, Ren W, Qin Z, Zhang X, Yuan W, Wang J, Yu W, et al: MiR-590-5p, a density-sensitive microRNA, inhibits tumorigenesis by targeting YAP1 in colorectal cancer. Cancer Lett 399: 53-63, 2017.

27. Antonov AV: BioProfiling.de: Analytical web portal for high-throughput cell biology. Nucleic Acids Res 39: W323-W327, 2011.

28. Amelio I, Tsvetkov PO, Knight RA, Lisitsa A, Melino G and Antonov AV: SynTarget: An online tool to test the synergetic effect of genes on survival outcome in cancer. Cell Death Differ 23: 912, 2016.
29. Bray F, Ren JS, Masuyer E and Ferlay J: Global estimates of cancer prevalence for 27 sites in the adult population in 2008. Int J Cancer 132: 1133-1145, 2013.

30. Ferlay J, Shin HR, Bray F, Forman D, Mathers C and Parkin DM: Estimates of worldwide burden of cancer in 2008: GLOBOCAN 2008. Int J Cancer 127: 2893-2917, 2010.

31. Ponting CP, Oliver PL and Reik W: Evolution and functions of long noncoding RNAs. Cell 136: 629-641, 2009.

32. He X, Ou C, Xiao Y, Han Q, Li H and Zhou S: LncRNAs: Key players and novel insights into diabetes mellitus. Oncotarget 8: 71325-71341, 2017

33. Ou C and Li G: Long non-coding RNA TUG1: A novel therapeutic target in small cell lung cancer. J Thorac Dis 9: E644-E645, 2017.

34. Prensner JR and Chinnaiyan AM: The emergence of IncRNAs in cancer biology. Cancer Discov 1: 391-407, 2011.

35. Schmitt AM and Chang HY: Long noncoding RNAs in cancer pathways. Cancer Cell 29: 452-463, 2016.

36. $\mathrm{Ou} \mathrm{C}$ and $\mathrm{Li} \mathrm{G}$ : Exosome-transmitted lncARSR: A novel therapeutic target in renal cancer. Transl Cancer Res 6, 656-657, 2017.

37. Yin DD, Liu ZJ, Zhang E, Kong R, Zhang ZH and Guo RH Decreased expression of long noncoding RNA MEG3 affects cell proliferation and predicts a poor prognosis in patients with colorectal cancer. Tumour Biol 36: 4851-4859, 2015.

38. Yin D, He X, Zhang E, Kong R, De W and Zhang Z: Long noncoding RNA GAS5 affects cell proliferation and predicts a poor prognosis in patients with colorectal cancer. Med Oncol 31: 253, 2014.

39. Zheng HT, Shi DB, Wang YW, Li XX, Xu Y, Tripathi P, Gu WL Cai GX and Cai SJ: High expression of lncRNA MALAT1 suggests a biomarker of poor prognosis in colorectal cancer. Int J Clin Exp Pathol 7: 3174-3181, 2014.

40. Sun J, Ding C, Yang Z, Liu T, Zhang X, Zhao C and Wang J: The long non-coding RNA TUG1 indicates a poor prognosis for colorectal cancer and promotes metastasis by affecting epithelial-mesenchymal transition. J Transl Med 14: 42, 2016.

41. Svoboda M, Slyskova J, Schneiderova M, Makovicky P, Bielik L, Levy M, Lipska L, Hemmelova B, Kala Z, Protivankova M, et al: HOTAIR long non-coding RNA is a negative prognostic factor not only in primary tumors, but also in the blood of colorectal cancer patients. Carcinogenesis 35: 1510-1515, 2014

42. Takahashi Y, Sawada G, Kurashige J, Uchi R, Matsumura T, Ueo H, Takano Y, Eguchi H, Sudo T, Sugimachi K, et al: Amplification of PVT-1 is involved in poor prognosis via apoptosis inhibition in colorectal cancers. Br J Cancer 110: 164-171, 2014.

43. Li Q, Dai Y, Wang F and Hou S: Differentially expressed long non-coding RNAs and the prognostic potential in colorectal cancer. Neoplasma 63: 977-983, 2016.

44. Colombo T, Farina L, Macino G and Paci P: PVT1: A rising star among oncogenic long noncoding RNAs. Biomed Res Int 2015: $304208,2015$.

45. Huang C, Liu S, Wang H, Zhang Z, Yang Q and Gao F: LncRNA PVT1 overexpression is a poor prognostic biomarker and regulates migration and invasion in small cell lung cancer. Am J Transl Res 8: 5025-5034, 2016.

46. Shen CJ, Cheng YM and Wang CL: LncRNA PVT1 epigenetically silences miR-195 and modulates EMT and chemoresistance in cervical cancer cells. J Drug Target 25: 637-644, 2017.

47. Guo K, Yao J, Yu Q, Li Z, Huang H, Cheng J, Wang Z and Zhu Y: The expression pattern of long non-coding RNA PVT1 in tumor tissues and in extracellular vesicles of colorectal cancer correlates with cancer progression. Tumour Biol 39: 1010428317699122, 2017.

This work is licensed under a Creative Commons Attribution-NonCommercial-NoDerivatives 4.0 International (CC BY-NC-ND 4.0) License. 